\title{
Material Contact and Environmental Effects on Vitamin A Fortified Vegetable Frying Oil
}

\author{
Sukmo Wening, ${ }^{1}$ Andayani, Dewi Yuliyanti, ${ }^{1}$ Latifun Jayanti, ${ }^{1}$ Isti Christianti, ${ }^{1}$ \\ Karyanto Mulyono ${ }^{1} \&$ Paul Wassell ${ }^{1} *$ \\ * Email: paul.wassell@sinarmas-agri.com \\ 1 Golden Agri-Resources (PT. SMART Tbk), Jakarta, Indonesia
}

\begin{abstract}
The impact of environmental parameters and time, on fortified vegetable oil, using typical commercial grade linear low density polyethylene (LLDPE) packaging during typical shelf-life conditions, were studied over long-term (12 months) to evaluate impact of temperature and illuminance (light: 100 to 150 lux and darkness <1 lux) on refined bleached deodorized palm olein (RBDPOL), fortified with vitamin A, without antioxidant. Results showed degradation of fortified RBDPOL with 70 ppm vitamin A was approximately $19 \%$ at $18-22^{\circ} \mathrm{C}$ in Light, $18 \%$ at $18-22^{\circ} \mathrm{C}$ in Darkness, $38 \%$ at $32-33^{\circ} \mathrm{C}$ in light and $24 \% 32-33{ }^{\circ} \mathrm{C}$ in darkness. $\mathrm{A}$ similar trend was observed at $45 \mathrm{ppm}$ vitamin A. Irrespective of the contact packaging material, a prolonged exposure at elevated temperature significantly impacts vitamin A depletion.
\end{abstract}

Key words --- Fortified, Illuminance, Packaging, Palm oil, Shelf-life, Stability, Vitamin A.

\section{INTRODUCTION}

Achieving healthy sustainable food systems requires large reductions in food loss and waste, together with improved food production practices [1]. Within this context, from 2012, several nations adopted fortification policies to include vegetable oil as a fortified delivery mechanism [2, 3]. Recently, several food manufacturers, have studied fortification of palm based vegetable cooking oil with vitamin A $[4,5]$.

Edible oil fortification, is a potentially useful means of expanding the present reach of vitamin A [6] But, stability is shown to be problematic in some environments [8, 5]. Factors such as acidity, moisture, ultraviolet light, oxygen and heat are well established causes [9 -13]. Clearly, given new global challenges of delivering sustainable food systems, attention should also be considered, not only for the food system itself, but also for the total delivery mechanism, which may be derived from natural materials used to protect the food system [1]. Undeniably, there is still continued interest to establish the most ideal methods for determining the most suitable environmental conditions and packaging materials [14] for long-term vitamin stabilisation [15, 16]. A recent study [5], noted several effects from choice of packaging material, to protect vitamin A and noted beneficial effects of Polyethylene Terepthalate (PET) vs transparent nylon, due to improved gas transmission and water vapour permeance. Opaque Nylon improved vitamin A retention, vs transparent PET, because vitamin A is more accute to UV light [17] than oxygen attack [18, 19].

Although, others [5] previously considered the effects of differing packaging materials on vitamin A degradation, the longest experimental design that we are aware of was only for six months [6]. No literature exists to explain the effect of illuminance on fortified vegetable oil using commercial packaging materials beyond six months in typical environmental shelf-life conditions. Consequently, this study is a continuation and update of previous studies at several isothermal conditions using normal commercial food grade packaging. Refined Bleached Deodorised Palm Olein (RBDPOL) was used and preferred for its superior oxidative stability. Antioxidants were excluded [20, 5, 6].

\section{MATERIALS AND METHOD}

Vegetable oil, RBDPOL, was supplied by PT. SMART, Tbk, Indonesia, with the following parameters: Iodine Value: 60.67 \pm 0.15 (AOCS Cd 1b-87); Peroxide Value: $0.5 \pm 0.02$ (AOCS Cd 8-53); Free Fatty Acid: $0.04 \pm 0.00$ (AOCS Ca 5a-40); Moist \& impurities: 0.05 \pm 0.01 (AOCS Ca 2b-38); Colour: 2.2 \pm 0.01 (AOCS Cc 13e-92)

Vitamin A 1.0 mio IU, was supplied from DSM Singapore. Specification as follows: Peroxide Value, meq kg-1 (Max. 10.0), Acid value, mg KOH g-1 (Max. 2.0), Assay (1.0-1.1 mio IU g-1).

Described previously [5, 6], preparations of RBDPOL and vitamin A 1.0 mio IU (retinyl palmitate) were assembled in laboratory conditions at concentrations of 70 IU g-1 and 45 IU g-1, at two temperature ranges; first 18$22^{\circ} \mathrm{C}$, and $32-33^{\circ} \mathrm{C}$, for a total duration of twelve months $[21,22,25]$.

Analysis of Iodine Value (IV), Peroxide value (PV), Free fatty acid (FFA), Moisture \& Impurities (M\&I), Colour 
and illuminance were determined by the same methods described above and previous [6].

Quantification of vitamin $A$ by high performance liquid chromatography (HPLC)

The vitamin A was determined by liquid chromatography method [26]. Standardized vitamin A (Retinyl palmitate) was supplied from Supelco-Sigma Aldrich (purity 93.8\%). HPLC determination is described previously [6]

\section{Statistical analysis}

Statistical analysis was first examined using a combination of Student t-test to determine uncertainty [27]. Final analysis was carried out using analysis of variance $(\alpha$ $=0.05$ ) with MiniTab v.16.1.1 software.

\section{RESULTS AND DISCUSSION}

\section{Calibration of Vitamin A}

A calibration of vitamin A level concentration (IU/g) is shown previously in [5]. Calculations show corellation between peak area obtained from analysis using HPLC and regression of linearity.

\section{Effect of temperature and illuminance on vitamin $A$ stability}

Figures 1 and 2, show the impact of both temperature and illuminance (light: 100 to 150 lux and darkness <1 lux) during storage of fortified RBDPOL with Vitamin A (70 and $45 \mathrm{ppm}$ ). Analysis after twelve (12) months monitoring, showed that degradation of RBDPOL fortified with $70 \mathrm{ppm}$ vitamin A was approximately $19 \%$ at $18-22^{\circ} \mathrm{C}$ in Light, $18 \%$ at $18-22^{\circ} \mathrm{C}$ in Dark, $38 \%$ at $32-33^{\circ} \mathrm{C}$ in light and $24 \%$ at $32-$ $33^{\circ} \mathrm{C}$ in dark. A similar trend was observed in RBDPOL fortified with $45 \mathrm{ppm}$ vitamin $\mathrm{A}$, for the same time period; after 12 months, the vitamin $\mathrm{A}$ had degraded by approximately $24 \%$ at $18-22^{\circ} \mathrm{C}$ in light, $16 \%$ at $18-22^{\circ} \mathrm{C}$ in dark, $38 \%$ at $32-33^{\circ} \mathrm{C}$ in light and $27 \%$ at $32-33^{\circ} \mathrm{C}$ in darkness.

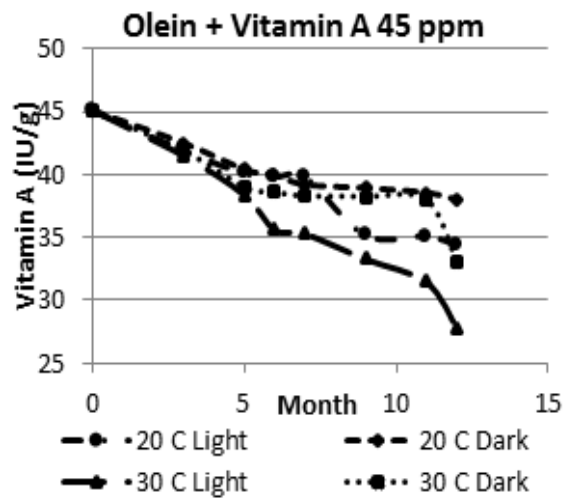

Fig. 1 Impact of temperature and illuminance (light: 100 to 150 lux and darkness $<1$ lux) during storage of fortified RBDPOL

(45 ppm vitamin A)

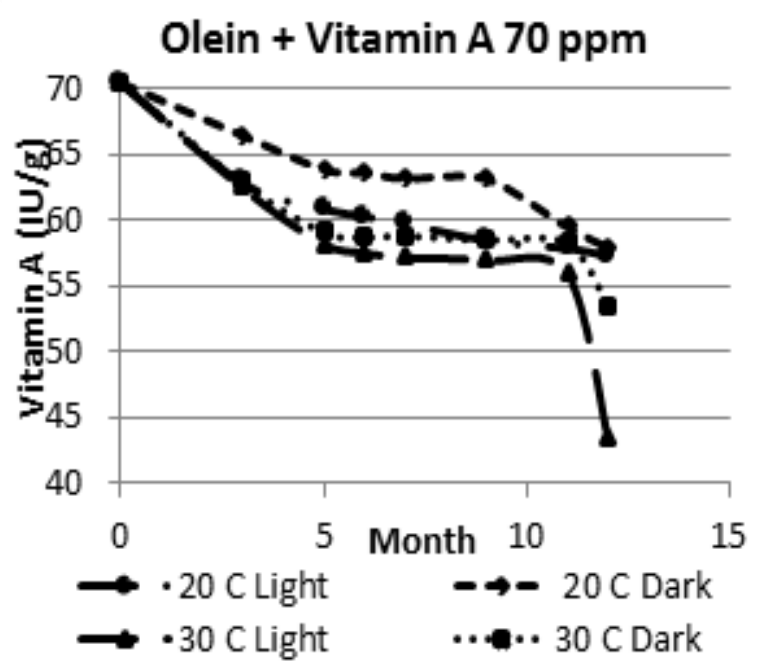

Fig. 2 Impact of temperature and illuminance (light: 100 to 150 lux and darkness $<1$ lux) during storage of fortified RBDPOL (70 ppm vitamin A)

The data also shows the effect of temperature on the degradation of vitamin A during storage. A temperature of $32-33^{\circ} \mathrm{C}$ giving higher Vitamin A degradation versus 18$22^{\circ} \mathrm{C}$. This result confirms similar findings on temperature impact of vitamin A degradation in [13]. Secondly, although test samples did not have direct exposure to natural sunlight, this observation shows the effect of vitamin A fortified RBDPOL to direct light exposure (100 to 150 lux) or darkness ( $<1$ lux). Light (100 to 150 lux) conditions, will accelerate vitamin A degradation in RBDPOL faster than if $<1$ Lux condition. Prolonged exposure of RBDPOL vegetable oil to illuminance does impact on vitamin $\mathrm{A}$ depletion

Table 4, shows that temperature storage is probably contributing the biggest impact to degradation of vitamin A. After twelve (12) months monitoring, all samples stored at $18-22^{\circ} \mathrm{C}$ had vitamin A degradation of approximately 10$15 \%$. Whereas, all samples stored at $32-33^{\circ} \mathrm{C}$ degraded by approximately $16-22 \%$. These data confirm similar findings [5].

\section{Correlation of $P V$ value to vitamin A degradation}

As described previously [5], for vitamin A fortification studies, the peroxide value (PV) was monitored to acceptable level of 10 meq kg-1 [20]. Figures 3 and 4 show the effect of peroxide (PV) to decrease vitamin A. Linearity was observed for $45 \mathrm{ppm}(\mathrm{y}=-0.3106 \mathrm{x}+43.035)$ and $70 \mathrm{ppm}(\mathrm{y}=-0.603 \mathrm{x}+67.149)$. This confirms a similar tendency noted by others for a compound decrease of vitamin $A$ in oxidized oil [5, 28, 29]. 


\section{MONTH}

\begin{tabular}{|c|c|c|c|c|c|c|c|c|c|c|c|c|c|c|c|c|c|c|c|c|c|c|c|c|}
\hline \multirow{2}{*}{ 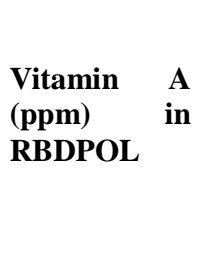 } & \multirow{2}{*}{$\begin{array}{l}\text { Storage } \\
\text { condition }\end{array}$} & \multicolumn{3}{|c|}{0} & \multicolumn{2}{|c|}{3} & \multicolumn{3}{|c|}{5} & \multicolumn{3}{|c|}{6} & \multicolumn{3}{|c|}{7} & \multicolumn{3}{|c|}{9} & \multicolumn{3}{|c|}{11} & \multicolumn{3}{|c|}{12} \\
\hline & & Vit $A^{\Delta}$ & $\mathbf{P V}^{\Delta \Delta}$ & Vit A & PV & $\begin{array}{c}\text { Vit A \% } \\
\text { Degraded }\end{array}$ & Vit $A$ & PV & $\begin{array}{c}\text { Vit A \% } \\
\text { Degraded }\end{array}$ & Vit A & PV & $\begin{array}{c}\text { Vit A \% } \\
\text { Degraded }\end{array}$ & Vit A & PV & $\begin{array}{c}\text { Vit A \% } \\
\text { Degraded }\end{array}$ & Vit A & PV & $\begin{array}{c}\text { Vit A \% } \\
\text { Degraded }\end{array}$ & Vit A & PV & $\begin{array}{c}\text { Vit A \% } \\
\text { Degraded }\end{array}$ & Vit A & PV & $\begin{array}{c}\text { Vit A \% } \\
\text { Degraded }\end{array}$ \\
\hline 70ppm (Light*) & & $\begin{array}{c}70.5 \pm \\
0.36\end{array}$ & $\begin{array}{l}0.5 \pm \\
0.01\end{array}$ & $\begin{array}{c}62.9 \pm \\
0.45\end{array}$ & $\begin{array}{l}5.9 \pm \\
0.06\end{array}$ & 11 & $\begin{array}{c}60.9 \pm \\
0.11\end{array}$ & $\begin{array}{l}9.1 \pm \\
0.06\end{array}$ & 14 & $\begin{array}{c}60.3 \pm \\
0.03\end{array}$ & $\begin{array}{l}10.1 \pm \\
0.05\end{array}$ & 15 & $\begin{array}{c}59.8 \pm \\
0.06\end{array}$ & $\begin{array}{c}11.1 \pm \\
0.15\end{array}$ & 15 & $\begin{array}{c}58.5 \pm \\
0.13\end{array}$ & $\begin{array}{c}14.9 \pm \\
0.14\end{array}$ & 17 & $\begin{array}{c}57.9 \pm \\
0.31\end{array}$ & $\begin{array}{l}20.5 \pm \\
0.04\end{array}$ & 18 & $\begin{array}{c}57.3 \pm \\
0.78\end{array}$ & $\begin{array}{c}21.6 \pm \\
0.01\end{array}$ & 19 \\
\hline 70ppm (Dark\#) & & $\begin{array}{c}70.5 \pm \\
0.36\end{array}$ & $\begin{array}{l}0.5 \pm \\
0.01\end{array}$ & $\begin{array}{c}66.3 \pm \\
0.45\end{array}$ & $\begin{array}{l}0.9 \pm \\
0.02\end{array}$ & 6 & $\begin{array}{c}63.8 \pm \\
0.11\end{array}$ & $\begin{array}{l}2.5 \pm \\
0.05\end{array}$ & 9 & $\begin{array}{c}63.6 \pm \\
0.20\end{array}$ & $\begin{array}{l}3.1 \pm \\
0.05\end{array}$ & 10 & $\begin{array}{c}63.2 \pm \\
0.01\end{array}$ & $\begin{array}{l}3.6 \pm \\
0.07\end{array}$ & 10 & $\begin{array}{c}63.2 \pm \\
0.08\end{array}$ & $\begin{array}{l}8.3 \pm \\
0.08\end{array}$ & 10 & $\begin{array}{c}59.5 \pm \\
1.39\end{array}$ & $\begin{array}{c}11.6 \pm \\
0.25\end{array}$ & 16 & $\begin{array}{c}58.0 \pm \\
0.55\end{array}$ & $\begin{array}{c}12.22 \pm \\
0.66\end{array}$ & 18 \\
\hline 45ppm (Light) & $18-22^{\circ} \mathrm{C}$ & $\begin{array}{c}45.1 \pm \\
1.01\end{array}$ & $\begin{array}{c}0.5 \pm 0 . \\
02\end{array}$ & $\begin{array}{c}42.0 \pm \\
1.26\end{array}$ & $\begin{array}{l}5.6 \pm \\
0.02\end{array}$ & 7 & $\begin{array}{c}40.1 \pm \\
0.08\end{array}$ & $\begin{array}{c}10.6 \pm \\
0.06\end{array}$ & 11 & $\begin{array}{c}39.8 \pm \\
0.14\end{array}$ & $\begin{array}{c}11.3 \pm \\
0.06\end{array}$ & 13 & $\begin{array}{c}39.8 \pm \\
0.06\end{array}$ & $\begin{array}{c}11.2 \pm \\
0.18\end{array}$ & 12 & $\begin{array}{c}35.1 \pm \\
0.05\end{array}$ & $\begin{array}{c}12.5 \pm \\
0.05\end{array}$ & 22 & $\begin{array}{c}35.1 \pm \\
0.13\end{array}$ & $\begin{array}{c}16.16 \pm \\
0.01\end{array}$ & 22 & $\begin{array}{c}34.4 \pm \\
0.05\end{array}$ & $\begin{array}{c}18.1 \pm \\
0.60\end{array}$ & 24 \\
\hline 45ppm (Dark) & & $\begin{array}{c}45.1 \pm \\
1.01\end{array}$ & $\begin{array}{l}0.5 \pm \\
0.02\end{array}$ & $\begin{array}{c}42.5 \pm \\
0.01\end{array}$ & $\begin{array}{l}0.8 \pm \\
0.06\end{array}$ & 6 & $\begin{array}{c}40.4 \pm \\
0.37\end{array}$ & $\begin{array}{l}2.4 \pm \\
0.01\end{array}$ & 10 & $\begin{array}{c}39.9 \pm \\
0.03\end{array}$ & $\begin{array}{l}2.2 \pm \\
0.05\end{array}$ & 13 & $\begin{array}{c}39.2 \pm \\
0.05\end{array}$ & $\begin{array}{l}2.7 \pm \\
0.02\end{array}$ & 13 & $\begin{array}{c}38.9 \pm \\
0.10\end{array}$ & $\begin{array}{l}6.6 \pm \\
0.13\end{array}$ & 14 & $\begin{array}{c}38.5 \pm \\
0.08\end{array}$ & $\begin{array}{l}11.1 \pm \\
0.02\end{array}$ & 15 & $\begin{array}{c}38.0 \pm \\
0.23\end{array}$ & $\begin{array}{l}8.8 \pm \\
0.03\end{array}$ & 16 \\
\hline 70ppm (Light) & & $\begin{array}{c}70.5 \pm \\
0.36\end{array}$ & $\begin{array}{l}0.5 \pm \\
0.01\end{array}$ & $\begin{array}{c}62.5 \pm \\
1.16\end{array}$ & $\begin{array}{l}9.2 \pm \\
0.09\end{array}$ & 11 & $\begin{array}{c}58.3 \pm \\
0.06\end{array}$ & $\begin{array}{c}14.4 \pm \\
0.04\end{array}$ & 17 & $\begin{array}{c}57.6 \pm \\
0.16\end{array}$ & $\begin{array}{l}16.7 \pm \\
0.08\end{array}$ & 19 & $\begin{array}{c}57.2 \pm \\
0.06\end{array}$ & $\begin{array}{c}18.2 \pm \\
0.35\end{array}$ & 19 & $\begin{array}{c}56.9 \pm \\
0.31\end{array}$ & $\begin{array}{c}25.7 \pm \\
0.16\end{array}$ & 19 & $\begin{array}{c}55.9 \pm \\
0.23\end{array}$ & $\begin{array}{c}30.9 \pm \\
0.01\end{array}$ & 21 & $\begin{array}{c}43.5 \pm \\
2.18\end{array}$ & $\begin{array}{c}31.47 \pm \\
0.61\end{array}$ & 38 \\
\hline 70ppm (Dark) & & $\begin{array}{c}70.5 \pm \\
0.36\end{array}$ & $\begin{array}{l}0.5 \pm \\
0.01\end{array}$ & $\begin{array}{c}62.9 \pm \\
0.26\end{array}$ & $\begin{array}{l}9.2 \pm \\
0.12\end{array}$ & 11 & $\begin{array}{c}59.1 \pm \\
0.01\end{array}$ & $\begin{array}{c}11.5 \pm \\
0.06\end{array}$ & 16 & $\begin{array}{c}58.8 \pm \\
0.11\end{array}$ & $\begin{array}{l}15.7 \pm \\
0.06\end{array}$ & 17 & $\begin{array}{c}58.4 \pm \\
0.01\end{array}$ & $\begin{array}{l}16 \pm \\
0.18\end{array}$ & 17 & $\begin{array}{c}58.5 \pm \\
0.01\end{array}$ & $\begin{array}{c}23.2 \pm \\
0.20\end{array}$ & 17 & $\begin{array}{c}58.3 \pm \\
0.13\end{array}$ & $\begin{array}{c}25.9 \pm \\
0.13\end{array}$ & 17 & $\begin{array}{c}53.5 \pm \\
0.37\end{array}$ & $\begin{array}{c}26.7 \pm \\
0.03\end{array}$ & 24 \\
\hline 45ppm (Light) & $32-33^{\circ} \mathrm{C}$ & $\begin{array}{c}45.1 \pm \\
1.01\end{array}$ & $\begin{array}{l}0.5 \pm \\
0.02\end{array}$ & $\begin{array}{c}41.6 \pm \\
0.26\end{array}$ & $\begin{array}{c}8.51 \pm \\
0.13\end{array}$ & 8 & $\begin{array}{c}38.4 \pm \\
0.08\end{array}$ & $\begin{array}{l}15 \pm \\
0.02\end{array}$ & 15 & $\begin{array}{c}35.6 \pm \\
0.04\end{array}$ & $\begin{array}{c}18.3 \pm \\
0.01\end{array}$ & 22 & $\begin{array}{c}35.3 \pm \\
0.11\end{array}$ & $\begin{array}{c}18.9 \pm \\
0.36\end{array}$ & 22 & $\begin{array}{c}33.3 \pm \\
0.37\end{array}$ & $\begin{array}{c}26.0 \pm \\
0.01\end{array}$ & 26 & $\begin{array}{c}31.5 \pm \\
0.44\end{array}$ & $\begin{array}{c}33.0 \pm \\
0.16\end{array}$ & 30 & $\begin{array}{c}27.8 \pm \\
0.40\end{array}$ & $\begin{array}{c}36.3 \pm \\
0.23\end{array}$ & 38 \\
\hline 45ppm (Dark) & & $\begin{array}{c}45.1 \pm \\
1.01\end{array}$ & $\begin{array}{l}0.5 \pm \\
0.02\end{array}$ & $\begin{array}{c}41.9 \pm \\
0.75\end{array}$ & $\begin{array}{l}5.5 \pm \\
0.13\end{array}$ & 7 & $\begin{array}{c}39.0 \pm \\
0.02\end{array}$ & $\begin{array}{c}13.5 \pm \\
0.04\end{array}$ & 14 & $\begin{array}{c}38.6 \pm \\
0.12\end{array}$ & $\begin{array}{c}14.9 \pm 0 \\
.01\end{array}$ & 16 & $\begin{array}{c}38.3 \pm \\
0.30\end{array}$ & $\begin{array}{c}17.3 \pm \\
0.16\end{array}$ & 15 & $\begin{array}{c}38.3 \pm \\
0.08\end{array}$ & $\begin{array}{c}22.2 \pm \\
0.04\end{array}$ & 15 & $\begin{array}{c}38.0 \pm \\
0.08\end{array}$ & $\begin{array}{c}28.4 \pm \\
0.04\end{array}$ & 16 & $\begin{array}{c}33.03 \pm \\
0.34\end{array}$ & $\begin{array}{c}=30.2 \pm \\
0.11\end{array}$ & 27 \\
\hline
\end{tabular}

\# Dark $=<1$ Lux

$* *$ Light $=100$ to $150 \mathrm{~L}$

${ }^{\Delta}$ Vitamin A IU g-1

${ }^{\Delta \Delta}$ PV meq/kg

31 | Wening, et al (2020) 
Table 4, shows that temperature storage is probably contributing the biggest impact to degradation of vitamin A. After twelve (12) months monitoring, all samples stored at $18-22^{\circ} \mathrm{C}$ had vitamin A degradation of approximately $10-$ $15 \%$. Whereas, all samples stored at $32-33^{\circ} \mathrm{C}$ degraded by approximately $16-22 \%$. These data confirm similar findings [5].

\section{Correlation of $P V$ value to vitamin A degradation}

As described previously [5], for vitamin A fortification studies, the peroxide value (PV) was monitored to acceptable level of 10 meq kg-1 [20]. Figures 3 and 4 show the effect of peroxide (PV) to decrease vitamin A. Linearity was observed for $45 \mathrm{ppm}(\mathrm{y}=-0.3106 \mathrm{x}+43.035)$ and $70 \mathrm{ppm}(\mathrm{y}=-0.603 \mathrm{x}+67.149)$. This confirms a similar tendency noted by others for a compound decrease of vitamin A in oxidized oil [5, 28, 29].

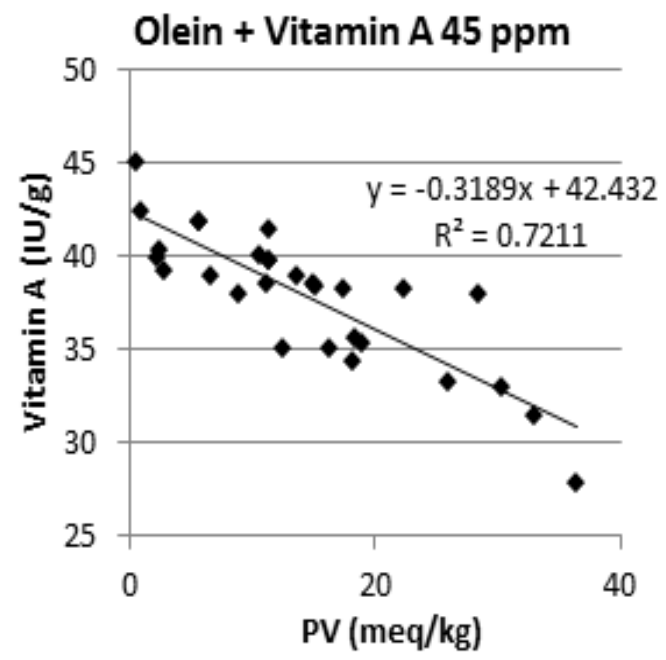

Fig. 3 Effect of PV during storage of fortified RBDPOL (45 ppm vitamin $\mathrm{A}$ )

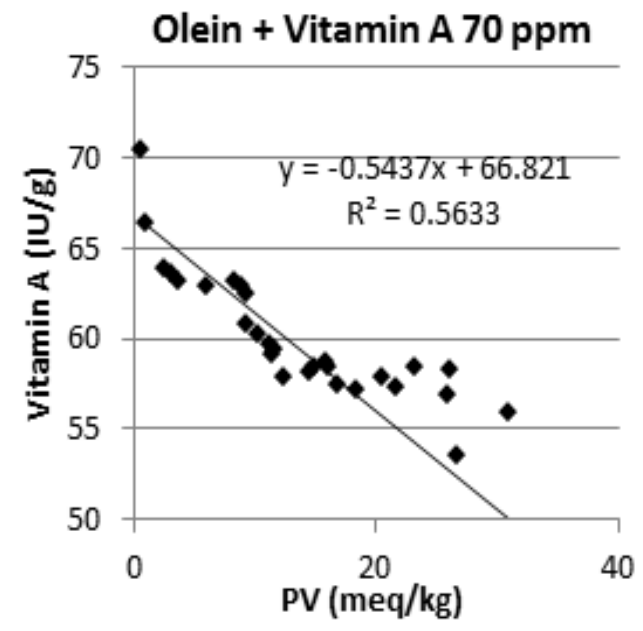

Fig. 4 Effect of PV during storage of fortified RBDPOL (70 ppm vitamin $\mathrm{A}$ )

This more extensive study validates previous research $[5,6]$ on the impact of environmental effects of fortified vegetable frying oil, during extensive storage life periods. These results show that cooler storage conditions, low illuminance or low UV light adsorbent packaging could aid to minimize excessive vitamin A depletion during distribution and storage [13, 15, 30]. Our results further suggest the benefits in selecting suitable contact packaging materials, which are able to minimize illuminance from natural or artificial UV light $[16,5]$, together with optimal storage temperatures to maximize vitamin A retention. Outcomes from this study may be of importance for food product developers, when fortified vegetable products are frequently sent via food aid programs to localities having insufficient storage controls [30].

\section{CONCLUSION}

After 12 months monitoring, the degradation of vitamin A was above $15 \%$ at the lowest temperature and $\sim 38 \% \pm$ at the highest temperature. This confirms previous findings [5], that fortification $45 \mathrm{IU}$ g-1 in vegetable frying oil is likely insufficient to maintain the Vitamin A level above 20 IU g-1 over entire storage life.

The effect of prolonged storage at elevated temperatures has a vivid effect. Cooler condition $\left(18-22^{\circ} \mathrm{C}\right)$, retained significantly more vitamin $\mathrm{A}$ vs samples stored at higher $\left(31-33^{\circ} \mathrm{C}\right)$ temperatures [5]. This study further reinforces the advantage of storing fortified oil in suitable low illuminance, away from direct light. Hence, controlled storage will help retain maximum vitamin A concentration [15].

This study excluded antioxidants to avoid distortion of results. Hence, a next step from these findings is to understand the effect of antioxidant addition; and additionally, the effect of new recycled or biodegradable food contact materials on fortified vegetable oil based systems.

\section{ACKNOWLEDGEMENTS}

Thank you to Golden Agri Resources (PT. SMART Tbk) for supporting publication of this research.

\section{REFERENCES}

[1]. Willett, W. Rocktröm, J. Loken, B. et al. (2019). Food in the Anthropocene: the EAT-Lancet Commission on healthy diets from sustainable food systems. http://dx.doi.org/10.1016/S01406736(18)31788-4

[2]. Global Alliance for Improved Nutrition (GAIN) (2010). Fortification of vegetable oil in Indonesia. www.gainhealth.org

[3]. Riskesdas (Riset kesehatan dasar) (2010). National report on basic health research by national institute of health research and development. Indonesia: Ministry of Health.

[4]. Soekirman, Soekarjo, D., Martianto, D., Laillou, A. \& Moenchpfanner, R. (2012). Fortification of Indonesian unbranded vegetable oil: public - private initiative, from pilot to large scale. Food and Nutrition Bulletin, 33, S301-S302.

[5]. Silalahi, D. K., Yuliyanti, D. , da Silva, M. , Christianti, I. , Mulyono, K. and Wassell, P. (2017), The stability of vitamin A in fortified palm olein during extended storage and thermal treatment. Int J Food Sci Technol, 52: 1869-1877. doi:10.1111/ijfs.13462. 
[6]. Pradipta, A. N. G., Andayani, S. W., Yulyianti, D., Jayanti, L., Silalahi, D. K. N., Christianti, I., Mulyono, K., \& Wassell, P. (2018). The Environmental effects on fortified palm olein in the Anthropocene. Indonesian Food Science \& Technology Journal, 2(1), 21-25. https://doi.org/10.22437/ifstj.v2i1.6421

[7]. FAO/WHO (2004). Vitamin and mineral requirements in human nutrition, 2nd ed. Report of a joint FAO/WHO expert consultation, Bangkok. Gwanfogbe, P.N., Chambers, E.I., Martin, G., Fotso, M., \& Smith.

[8]. FAO/WHO (2006). Guidelines on food fortification with micronutrients. Allen, L, Benoist, Bruno de, Dary, O., et al.

[9]. Atwood, S.G., Sanghvi, T.G., Sharma, V. \& Carolan, N. (1995). Stability of vitamin A in fortified vegetable oil and corn soy blend used in child feeding programs in India. Journal of Food Composition and Analysis, 8, 32-44.

[10]. Farhang, B and Nikoopur, H. (2004) the effect of light and temperature on stability of vitamin A in the fortified vegetable oils (hydrogenated and nonhydrogenated). Asia pacific journal of clinical nutrition 2004.

[11]. Combs Jr, G.F. (2008). The Vitamins: Fundamental aspect in nutrition and health. Elsevier.inc. $3^{\text {rd }}$ ed.

[12]. Hariyadi, P. (2009). Technical aspects of oil fortification with vitamin A. Industrial Role of Cooking Oil Fortification with Vitamin A in Indonesia. Southeast Asian Food \& Agricultural Science \& Technology (SEAFAST) Center, Bogor Agricultural University, BOGOR, Indonesia. April 21, 2009.

[13]. Andarwulan, N., Gitapratiwi, D., Laillou, A. et al. (2014). Quality of vegetable oil prior to fortification is an important criteria to achieve a health impact. Nutrients, 6, 5051-5060.

[14]. Siracusa, V. (2012). Food packaging permeability behaviour: a report. International Journal of Polymer Science, 1-11.

[15]. Dwyer, J.T., Wiemer, K.L., Dary, O. et al. (2015). Fortification and health: challenges and opportunities. Advances in Nutrition, 6, 124131. https://doi.org/10.3945/an.114.007443

[16]. Barrett, A. H., Richardson, M. J., Froio, D. F., O'Connor, L. F., Anderson, D. J. and Ndou, T. V. (2018). Long-Term Vitamin Stabilization in Low Moisture Products for NASA: Techniques and Three-Year Vitamin Retention, Sensory, and Texture Results. Journal of Food Science, 83: 2183-2190. doi:10.1111/17503841.14218

[17]. Carlotti, M.E., Rossatto, V., Gallarate, M., et al. (2004). Vitamin A palmitate photostability and stability overtime. J. Cosmet. Sci., 55, $233-252$
[18]. Andarwulan, N., Muhammad, G.N., Agista, A.Z. et al. (2016). Photo oxidation stability of palm oil fortified by red palm oil. Journal Teknol. and Food Industry, 27, 31-39. ISSN: 1979-7788.

[19]. Hemery, Y.M., Fontan, L., Moench-Pfanner, R. et al. (2015). Influence of light exposure and oxidative status on the stability of vitamins A and D3 during the storage of fortified soybean oil. Food Chemistry, 184, 90-98.

[20]. Pignitter, M., Hernler, N., Zaunschirm, M. et al. (2016). Evaluation of palm oil as a suitable vegetable oil for vitamin a fortification programs. Nutrients, 8, 378. doi:10.3390/nu8060378.

[21]. Howell, R., Rosario, L., Bula, A. (1997) Effects of indoor relative humidity on refrigerated display case performance. In: Proceedings of CLIMA 2000, Brussels, Belgium.

[22]. BMKG (Badan Meteorolgi, Klimatologi, dan Geofisika) (2017). Minimum and Maximum temperature Tanjung Priok Jakarta at 1981-2010. http://web.meteo.bmkg.go.id/id/iklim (5 January 2018)

[23]. Japanese Industrial Standard (2006) JIS C 1609-1 Illuminance meters Part 1: General measuring instruments. ICS Code (Optical measuring instruments): 17.180.30. Pub: Japanese Standards Association.

[24]. CIBSE (2012). Lighting Guide 01: The Industrial Environment LG01. Chartered Institute of Building Service Engineers.

[25]. Bagriansky, J. \& Ranum, P. (1998). Vitamin A fortification of PL 480 vegetable oil. US Agency for International Development,1-29.

[26]. Association of Analytical Chemists (2011). AOAC official method 2001.13. Vitamin A (retinol) in foods, liquid chromatography, 19th ed., Chapter 45.1.

[27]. Birch, K. (2003). Estimating uncertainties in testing, measurement good practice guide No. 36. British Measurement and Testing Association. ISSN 1368-6550.

[28]. Pignitter, M., Dumhart, B., Gartner, S. et al. (2014a). Vitamin A is rapidly degraded in retinyl palmitate-fortified soybean oil stored under household conditions. Journal of Agricultural and Food Chemistry, 62, 7559-7566.

[29]. Pignitter, M., Stolze, K., Gartner, S. et al. (2014b). Cold fluorescent light as major inducer of lipid oxidation in soybean oil stored at household conditions for eight weeks. Journal of Agricultural and Food Chemistry, 62, 2297-2305.

[30]. Osendarp, S.J.M., Martinez, H., Garrett, G.S. et al. (2018). LargeScale Food Fortification and Biofortification in Low- and MiddleIncome Countries: A Review of Programs, Trends, Challenges, and Evidence Gaps. Food and Nutrition Bulletin. 39 (2) pp. 315-331 https://doi.org/10.1177/0379572118774229 